\title{
The role of antibodies to Bacillus anthracis and anthrax toxin components in inhibiting the early stages of infection by anthrax spores
}

\author{
Susan Welkos, ${ }^{1}$ Stephen Little, ${ }^{1}$ Arthur Friedlander, ${ }^{1}$ David Fritz ${ }^{2}$ \\ and Patricia Fellows ${ }^{1}$
}

Divisions of Bacteriology 1 and Pathology2, US Army Medical Research Institute of Infectious Diseases, Fort Detrick, Frederick, MD 21702-5011, USA
Author for correspondence: Susan Welkos. Tel: +1 301619 4930. Fax: +1 3016192152. e-mail:welkos@ncisun1.ncifcrf.gov

Vaccines which are efficacious against anthrax, such as the human vaccine, Anthrax Vaccine Absorbed (AVA), contain the protective antigen (PA) component of the anthrax toxins as the major protective immunogen. Although AVA protects against inhalational anthrax, the immune responses to and role in protection of PA and possibly other antigens have yet to be fully elucidated. Sera from animals immunized with a toxin-producing, unencapsulated live vaccine strain of Bacillus anthracis have been reported to have anti-spore activities associated with the antitoxin humoral response. The authors performed studies to determine whether anti-PA antibody (Ab)containing preparations stimulated spore uptake by phagocytes and suppressed the germination of spores in vitro. AVA- and PA-immune sera from several species enhanced the phagocytosis by murine peritoneal macrophages of spores of the virulent Ames and the Sterne vaccine strains. Antitoxin Abs appeared to contribute significantly, although not solely, to the enhanced uptake. Rabbit antisera to PA purified from either Sterne or a PA-producing pX01-cured recombinant, affinity-purified anti-PA IgG, and monkey antisera to AVA were used to assess the role of anti-PA Abs. Rabbit anti-PA Abs promoted the uptake of spores of the PA-producing strains Sterne, Ames and RP42, a mutant of Sterne producing only PA, but not of the pX01- $\Delta$ Sterne-1 strain, $\triangle$ Ames strain, or RP4, a mutant of Sterne with deletions in the loci encoding PA and the oedema factor (EF) toxin component and producing only the lethal factor toxin component. Rabbit anti-PA and monkey anti-AVA Abs also significantly inhibited spore germination in vitro compared to preimmune serum or medium. Spore-associated proteins recognized by anti-PA Abs were detected by electron microscopy and confirmed by immunoblotting of spore coat extracts. Thus, the anti-PA Ab-specific immunity induced by AVA has antispore activity and might have a role in impeding the early stages of infection with $B$. anthracis spores.

Keywords: antitoxin antibody, protective antigen, vaccines, phagocytosis

\section{INTRODUCTION}

The licensed human vaccine Anthrax Vaccine Absorbed (AVA), prepared from culture supernatants of a toxigenic, unencapsulated strain of Bacillus anthracis, protects animals against inhalational anthrax. Numerous

Abbreviations: AVA, Anthrax Vaccine Adsorbed; BHI, brain heart infusion; EF, (o)edema factor; $H$, heat-activated, ungerminated; HRP, horseradish peroxidase; LF, lethal factor; PA, protective antigen. animal studies have shown that the primary immunogen in AVA is the protective antigen (PA) component of the anthrax toxins (Ivins et al., 1996; Pitt et al., 1996, 1999). The mechanisms of protection induced by AVA remain unknown, although recent studies in rabbits have shown that protection is correlated with antibody ( $\mathrm{Ab}$ ) to PA (Pitt et al., 1999), and Ab to PA can confer passive protection on rabbits and guinea pigs (Little et al., 1997; S. F. Little and others, unpublished results). In other recent studies, Stepanov et al. (1996) reported that 
sera from animals vaccinated with a unencapsulated, toxigenic, live vaccine strain have two anti-spore activities that may be important during the early stages of infection. These are the inhibition of spore germination by antitoxin $\mathrm{Abs}$ and the stimulation of phagocytosis by rabbit peritoneal macrophages of spores by the $\operatorname{IgM}$ fraction of the immune sera. These findings suggest that the immunity induced by live anthrax vaccine strains might involve not only toxin-specific, but also sporespecific, activities. To determine the role of the antispore functions in vaccine-associated immunity, we characterized the effects of Abs to the toxin components, especially PA, and to non-toxin antigens on spore opsonization and germination.

\section{METHODS}

Bacterial strains and spore preparations. Strains of $B$. anthracis used included the virulent Ames strain, which harbours the plasmids encoding the major virulence antigens, the anthrax toxins (pX01) and the capsule (pX02); the unencapsulated $\left(\mathrm{pX} 02^{-}\right)$, toxigenic $\left(\mathrm{pX01}{ }^{+}\right)$Sterne vaccine strain; and plasmid-cured derivatives of them. The latter included ANR-1, a pX02-cured unencapsulated variant of Ames; and $\Delta$ Ames- 1 and $\Delta$ Sterne-1, toxin-negative, pX01cured isolates of Ames and Sterne, respectively. Two derivatives of Sterne strain 7702, RP4 and RP42, with mutations in the toxin component genes, were also kindly provided by $\mathrm{M}$. Mock (Pasteur Institute, Paris, France). The anthrax toxins consist of two proteins that interact non-covalently to produce functional toxin, either lethal toxin [lethal factor (LF) + PA] or oedema toxin [edema factor $(\mathrm{EF})+\mathrm{PA}]$. Mutant RP4 has deletions in the loci encoding PA and EF and produces only LF; RP42 has deletions in both the EF and LF genes and produces only the PA component (Pezard et al., 1991, 1993). All growth media for the latter two strains contained erythromycin $\left(10 \mu \mathrm{g} \mathrm{ml}^{-1}\right)$ and kanamycin $\left(20 \mu \mathrm{g} \mathrm{ml}^{-1}\right)$. Spores were prepared and purified from broth cultures of the strains containing at least $85 \%$ spores as described previously (Leighton \& Doi, 1971; Welkos et al., 1989). They were purified by centrifugation on density gradients of Hypaque-76 (Nycomed) and were washed, suspended and diluted in sterile water for injection (McGaw). The spores were activated by heating (Leighton \& Doi, 1971), germinated by incubation in brain heart infusion broth $(\mathrm{BHI})$ at $37^{\circ} \mathrm{C}$ and evaluated by phase microscopy.

Sera and antibody preparations. Immune sera from goats, guinea pigs, rabbits and monkeys were obtained from animals vaccinated with AVA, the Sterne strain, or purified PA. Antisera were also obtained from humans vaccinated with AVA. Rabbit antisera designated anti-St. PA and anti-rPA antisera were from animals hyperimmune to PA purified, respectively, from either the native Sterne strain or $\Delta$ Sterne-1 CR4 (CR4), a plasmid pX01-cured derivative of Sterne that carries the recombinant plasmid pPA102, consisting of the PA gene from Sterne cloned into plasmid pUB110 (Ivins \& Welkos, 1986; Worsham \& Sowers, 1999). The former had a mean reciprocal anti-PA $\operatorname{IgG}$ titre of $1.2 \times 10^{6}$ and the latter had a titre of $1.35 \times 10^{6}$, as determined using an antigencapture ELISA (Little \& Knudson, 1986). IgG was purified by Protein A column chromatography from rabbit anti-St. PA and anti-rPA sera and from normal rabbit serum (Pepper, 1990). The anti-PA IgG preparations had anti-PA ELISA titres greater than $10^{6}$. Sera collected from rabbits prior to vaccination (preimmune) and from non-immune animals, and the
IgG preparation purified from the latter, had mean anti-PA titres of $<50$. Monkey antisera were from animals inoculated intramuscularly with two doses of AVA (diluted $1 / 12 \cdot 5$ ), given $28 \mathrm{~d}$ apart, and were obtained 2 weeks after the second dose. Affinity-purified rabbit anti-PA IgG was obtained by chromatography of anti-rPA antisera over a PA antigen column followed by a Protein A column. Monoclonal antibodies (mAbs) that are specific for two major epitopes on the PA molecule were prepared as described previously (Pepper, 1990; Little \& Lowe, 1991; Little et al., 1988, 1996). The mAb PAI-2D5-1-1 recognizes an epitope between amino acids (aa) 581 and 601 and inhibits binding of LF to cell-bound PA; PA211-14B7-1-1 recognizes an epitope between aa 671 and 721 and blocks the C-terminal cell receptor site for PA.

\section{Preparation of macrophages and in vitro phagocytosis of} spores. Peritoneal exudate macrophages from mice were cultured for $3-5 \mathrm{~d}$ at $37^{\circ} \mathrm{C}$ in $5 \% \mathrm{CO}_{2}$ on coverslips in 24 -well plates, as described by Welkos et al. (1989). The cells were washed and infected with opsonized spores of $B$. anthracis (mean of $1 \times 10^{7}$ c.f.u. ml ${ }^{-1}$, with a usual m.o.i. of 5-10 and a range of 3-20). The spores were opsonized by incubation on ice for $30 \mathrm{~min}$ with immune serum or IgG, preimmune serum or IgG, or medium alone. They were then added to macrophages and the culture was incubated for $45 \mathrm{~min}\left(\right.$ at $37^{\circ} \mathrm{C}$ in $5 \%\left(\mathrm{CO}_{2}\right)$. The $45 \mathrm{~min}$ incubation period was the minimum time required to achieve maximal phagocytosis of spores by the $\mathrm{C} 3 \mathrm{H} / \mathrm{HeN}$ peritoneal macrophages, without observing significant spore clumping in the medium. The coverslips were washed ten times with HBSS (Hanks' Balanced Salts Solution) containing $10 \mathrm{mM}$ HEPES, pH $7 \cdot 0$ or $20 \mathrm{mM}$ PBS without $\mathrm{Ca}^{2+}$ or $\mathrm{Mg}^{2+}$ to remove unphagocytosed spores. The coverslips were then removed from the wells and further washed by rinsing sequentially in three $150 \mathrm{~mm}^{3}$ disposable sterile beakers (Falcon) prior to fixation and staining. Phagocytosis was measured by direct microscopic counts of samples stained with Wright-Giemsa and spore stains as described previously by Welkos et al. (1989). Specifically, the number of spores in $\geqslant 10$ fields, and a total of at least 100 macrophages, were counted on each of the duplicate coverslips per sample. All the macrophages in a field, whether infected or uninfected, were included. The mean number of spores per infected macrophage and the percentage of macrophages harbouring one or more organisms were calculated, and the data were expressed as the phagocytic index (PI), where PI = (mean no. of spores per infected macrophage $) \times(\%$ macrophages containing one or more spores).

Germination of serum-treated spores. Heat-activated, refractile ungerminated spores of $B$. anthracis strain Ames $\left(3 \times 10^{8}\right.$ spores) were incubated on ice for 30 min with serum or IgG diluted in Dulbecco's Minimal Essential Medium (DMEM). The pre-treated spores were then added to germination medium, usually $1 \%(\mathrm{v} / \mathrm{v}) \mathrm{BHI}$ in water, and incubated with shaking at $30^{\circ} \mathrm{C}$. At intervals, samples were removed to be read spectrophotometrically $\left(\right.$ at $\mathrm{OD}_{560}$ ), tested for heatresistance, or fixed in $4 \%$ formaldehyde for microscopy. An unstained aliquot of the latter was examined with phasecontrast microscopy and a second aliquot was stained with Gram's crystal violet and examined microscopically. The heat resistance of spores was determined by incubation of paired samples at $65^{\circ} \mathrm{C}$ or on ice for $30 \mathrm{~min}$; and plating dilutions on trypticase soy agar (TSA) plates for viable counts (Levinson \& Hyatt, 1966). The extent of germination was assessed as described previously by (1) decline in $\mathrm{OD}_{560}$; (2) loss of refractility by phase-contrast microscopy; (3) increase in stainability; and (4) loss of heat resistance (Levinson \& Hyatt, 1966). 

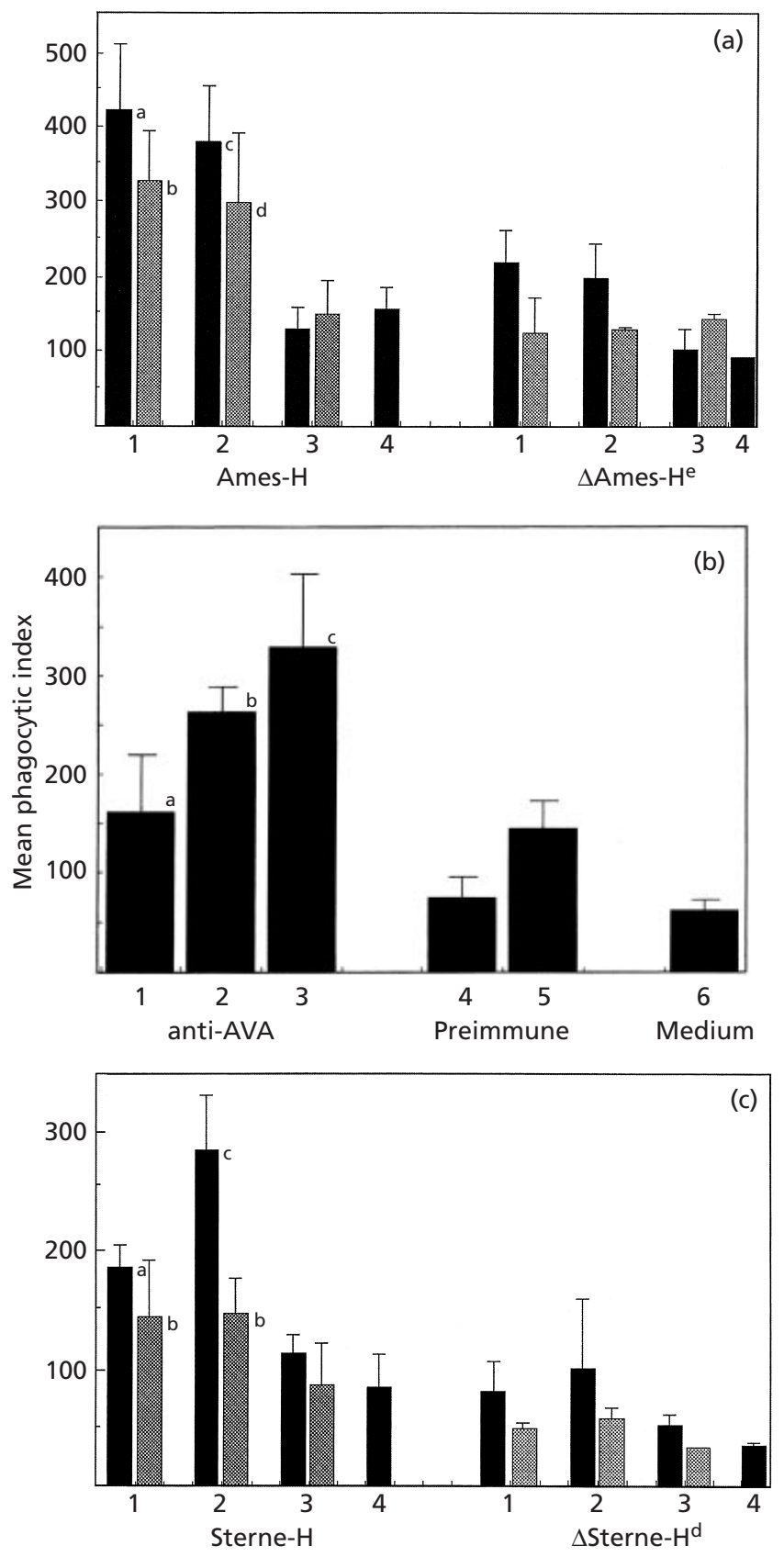

Fig. 1. Effect of anti-PA antisera and IgG on the phagocytosis of heat-activated $(\mathrm{H})$ ungerminated spores of the Ames and Sterne strains. Spores were opsonized by sera, IgG (data not shown) or medium alone before phagocytosis by macrophages and PI values were determined, as described in Methods. (a) Spores of Ames-H were opsonized by anti-rPA, rabbit antisera to recombinant PA purified from $\Delta$ Sterne-1 CR4 strain (1), antiSt. PA, rabbit antiserum to PA purified from the Sterne strain (2), preimmune serum (3), or medium alone (4). The black bars are the PI values for the $1 / 100$ dilutions of serum and the medium control; the hatched bars are data for the 1/400 dilutions. The data are the mean ( \pm SEM) of PI values from six to seven experiments for Ames $(H)$ and one for the pX01-cured derivative of Ames, $\Delta$ Ames- $1(\mathrm{H})$. ${ }^{\mathrm{a}} P=0.015$ vs preimmune serum $(1 / 100)$ and $P=0.023$ vs medium alone. ${ }^{b} P=0.053$ vs preimmune serum $(1 / 400)$ and $P=0.047$ vs medium. ${ }^{c} P=0.015$ vs preimmune serum $(1 / 100)$ and $P=0.025$ vs medium. ${ }^{d}$ Not significantly greater than PI for preimmune serum (1/400) or
Immunoelectron microscopy. Spores were fixed in $8 \%(\mathrm{v} / \mathrm{v})$ formaldehyde (Tousimis Research Co.), incubated for $7 \mathrm{~d}$ at $4{ }^{\circ} \mathrm{C}$, washed in PBS and processed as described previously (Fritz et al., 1995; Ezzell et al., 1990). Sections were incubated with rabbit anti-rPA antiserum and then goat anti-rabbit IgG conjugated with colloidal gold. They were poststained and examined in a Philips CM100 transmission electron microscope. Negative controls included samples with no primary antibody or with rabbit preimmune serum.

Spore extraction. Spores from which surface proteins were to be extracted were purified by density-gradient centrifugation and stored in sterile water for injection. Just before extraction, an aliquot containing approximately $4 \times 10^{9}$ $5 \times 10^{9}$ spores $\mathrm{ml}^{-1}\left(1 \times 10^{10}-3 \times 10^{10}\right.$ spores total $)$ was heatactivated by incubation at $65^{\circ} \mathrm{C}$ for $30 \mathrm{~min}$ and then chilled on ice. Samples of germinated spores were prepared by incubating the activated spores in BHI at $37^{\circ} \mathrm{C}$ until germinated. The spore suspensions were centrifuged and the pellets were suspended in a volume of extraction buffer equal to that of the original volume. The extraction buffer consisted of $0.1 \mathrm{M} \mathrm{DTT}, 0.5 \%$ (w/v) SDS, $0.1 \mathrm{M} \mathrm{NaCl}, \mathrm{pH} 10.0$ and was prepared as described previously (Vary, 1973). Small volumes of spores were placed as aliquots in microtubes $(0.6 \mathrm{ml}$ per tube $)$ and the tubes were incubated in a microtube incubator shaker (Eppendorf Thermomixer, Brinkman Instruments) at 1200 r.p.m. Larger volumes were aliquoted into $15 \mathrm{ml}$ plastic tubes and incubated horizontally in a tabletop shaker (LabLine) at 120 r.p.m. The suspensions were incubated for $2.5 \mathrm{~h}$ at $37^{\circ} \mathrm{C}$. The spores were then centrifuged in a microfuge for $5 \mathrm{~min}$ and the supernatants collected and filtered through $0 \cdot 2 \mu \mathrm{m}$ filters. These samples were dialysed, washed in MilliQ water and concentrated three- to fourfold by centrifugation in Centricon-3 or -10 concentrators (Amicon). These preparations were stored at $-70{ }^{\circ} \mathrm{C}$ until analysed by SDSPAGE.

Toxin neutralization assay. An in vitro colorimetric assay (Hansen et al., 1989) was used to determine the viability of J774A.1 cells exposed to lethal toxin in the presence of $\mathrm{Ab}$. Antiserum was preincubated with lethal toxin $\left(50 \mathrm{ng} \mathrm{PA} \mathrm{ml}^{-1}\right.$ with $40 \mathrm{ng} \mathrm{LF} \mathrm{ml} \mathrm{m}^{-1}$ ) for $1 \mathrm{~h}$ at $37^{\circ} \mathrm{C}$ before it was added to the cells. After 4 h, 3-(4,5-dimethylthiazol-2-yl)-2,5-diphenyltetra-

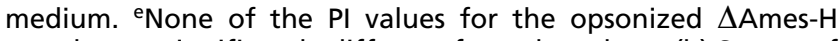
samples are significantly different from the others. (b) Spores of Ames- $\mathrm{H}$ were incubated with sera from three monkeys vaccinated with AVA (1, 2 and 3), serum from a naive normal control animal (4), preimmune serum from immune monkey 3 (5), or medium alone (6). All sera were assayed at a dilution of $1 / 100$ and the results expressed as described in (a). ${ }^{a}$ The PI for serum 1 was not significantly greater than that for the normal control serum $(4, P=0.12)$ or medium $(P=0.07)$. ${ }^{b}$ For serum 2 , $P=0.0005$ compared to the PI for the normal control (4) and $P$ $=0.025$ compared to the medium control. 'For serum $3, P=$ 0.05 compared to the $\mathrm{PI}$ for preimmune serum (5) and $P=0.016$ compared to the medium (6). (c) Spores of Sterne-H were opsonized by rabbit anti-rPA serum (1), anti-St. PA serum (2), preimmune serum (3), or medium alone (4); the $x$ axis samples and bar symbols are as described in panel (a). The data are the mean $( \pm$ SEM) of PI values from five experiments for Sterne-H and two to four experiments for the pX01-cured $\Delta$ Sterne- $H$, except that one experiment was run for the 1/400 dilution of preimmune serum. ${ }^{a} P=0.048$ vs preimmune serum $(1 / 100$ dilution) and $P=0.030$ vs medium control. ${ }^{b}$ Not significantly greater than $\mathrm{PI}$ for preimmune serum (1/400 dilution) or medium control. ${ }^{c} P=0.014$ vs preimmune serum $(1 / 100$ dilution) and $P=0.007$ vs medium control. ${ }^{\mathrm{d}}$ None of the $\mathrm{PI}$ values for the opsonized $\Delta$ Sterne samples are significantly different from the others. 
zolium bromide (MTT) was added and $2 \mathrm{~h}$ later the cells were lysed. Absorbance values at 570-690 nm were read, the titre was calculated by linear regression analysis (Instat, GraphPad Software), and expressed as the reciprocal of the dilution of antiserum that protected $50 \%$ of the J774A.1 cells.

SDS-PAGE and immunoblot analysis. Samples were electrophoresed on $10 \%$ Tricine gels (Novex) by SDS-PAGE under non-reducing conditions. The separated proteins were either visualized by silver stain or electrophoretically transferred to $0.45 \mu \mathrm{m}$ nitrocellulose membranes (Towbin et al., 1979). The membranes were blocked with $5 \%(\mathrm{w} / \mathrm{v})$ non-fat dry milk in PBS before incubating with rabbit anti-rPA serum, affinitypurified IgG prepared from the latter, a mixture containing two $\mathrm{mAb}$ ascitic fluids to PA (described above), or preimmune rabbit serum. The membranes were washed in PBS containing $0.1 \%(\mathrm{v} / \mathrm{v})$ Tween-20 before incubation in horseradish peroxidase (HRP) conjugated to goat antiserum against either rabbit or mouse IgG. Reactive components were visualized by enhanced chemiluminescence (ECL; Pierce).

Statistical analyses. The data were analysed by standard statistical methods [means, standard errors of the means (SEM), analysis of variance and unpaired Student's $t$-tests]. Macrophage phagocytosis values were expressed as the PI (described above). In germination inhibition experiments with the purified IgG from Ra anti-St. PA and monkey anti-AVA antisera, the data analysed were the mean values ( \pm SEM), for seven and four experiments, respectively, for spores treated with the dilution of purified IgG from the antiserum that maximally inhibited germination (compared to spores treated with identically diluted preimmune IgG or serum). In comparing groups, a $P$ value of $\leqslant 0.05$ was considered to indicate a significant difference.

\section{RESULTS}

\section{Stimulation of the phagocytosis of spores by immune sera and IgG}

The effects of immune sera on the phagocytosis of spores of the Ames strain by mouse peritoneal macrophages were studied. Antisera from several species (goat, monkey, guinea pig and rabbit), obtained by injecting AVA, PA, Sterne strain spores, or CR4 (the pX01-, PAproducing recombinant derivative of Sterne) enhanced phagocytosis compared to preimmune sera. Similar activity was observed using sera from humans injected with AVA. Antisera from guinea pigs inoculated with $\Delta$ Sterne-1 (a pX01 ${ }^{-}$derivative of the Sterne strain that does not produce PA) also enhanced phagocytosis compared to non-immune sera (data not shown), suggesting a role for Abs to non-toxin antigens as well as toxin-plasmid encoded ones. However, sera from some species were difficult to evaluate due to high background levels of phagocytosis. The latter appeared to result from spore aggregation by the sera (human) or from non-specific phagocytosis (goat, individual non-human primates; data not shown). Nevertheless, sera from rabbits vaccinated with $\mathrm{PA}$, and from most monkeys vaccinated with AVA, enhanced phagocytosis without significant serum-associated clumping or high background.

Pretreating heat-activated, ungerminated spores $(\mathrm{H})$ of Ames with rabbit antisera to PA purified from recombinant Sterne strain CR4 (Fig. 1a, sample 1) or from non- recombinant Sterne (Fig. 1a, sample 2) significantly enhanced the uptake of spores by the peritoneal macrophages over uptake by preimmune serum (Fig. 1a, sample 3). In contrast, the treatment of the pX01$\Delta$ Ames strain $(\Delta$ Ames- $H)$ with the antisera did not have a significant opsonizing effect on the spores (Fig. 1a). The phagocytosis-stimulating activity of sera from rabbits vaccinated with a toxigenic live vaccine was previously reported to be determined largely by the IgM fraction of the immune sera (Stepanov et al., 1996). However, we found that the $\operatorname{Ig} G$ fraction purified from the rabbit anti-St. PA antiserum also had a phagocytosisenhancing effect when compared to the uptake of spores pre-treated with comparable dilutions of preimmune $\operatorname{IgG}$ (data not shown). In addition to rabbit antisera, the sera from two of three monkeys vaccinated with the human AVA vaccine had phagocytosis-enhancing activities greater than those of preimmune or normal sera (Fig. 1b).

The immune-sera-mediated enhancement of phagocytosis was demonstrable for strains of $B$. anthracis in addition to Ames. Phagocytosis of ungerminated spores of the Sterne vaccine strain, but not of the pX01-cured $\Delta$ Sterne-1 strain, was increased by pretreatment with the anti-PA antisera (Fig. 1c). Although the macrophages were infected with the same concentrations of spores as used in the experiments depicted in Fig. 1(a), the lower PI values for the Sterne, compared to the Ames, strain, suggest that there may be strain-related quantitative differences in the extent of anti-PA sera-specific and non-specific phagocytosis. The basis of these differences requires further study.

Although antitoxin Abs appear to play a major role in the enhancement of spore phagocytosis, other factors might be involved. The existence of the latter was suggested, for instance, by the enhanced uptake of $\mathrm{pX} 01^{+}$spores by antisera elicited in guinea pigs by the pX01 ${ }^{-} \Delta$ Sterne-1 strain (data not shown), and by the incomplete correlation between anti-PA Ab titre and the phagocytosis-enhancing activity of monkey antisera. Although the antiserum obtained from AVA-vaccinated monkeys 1 and 3 had anti-PA titres of 12800 and 800, respectively, the antiserum from monkey 3 had more phagocytosis-enhancing activity than did that from monkey 1 (Fig. 1b). The toxin-neutralizing titres of the sera (1157 and 299, respectively, for monkeys 1 and 3) also did not correlate with the anti-spore activity of the sera.

\section{Inhibition of spore germination by immune sera and anti-PA $\lg G$}

Incubation in $\mathrm{BHI}$, or in $\mathrm{BHI}$ diluted in water to $2 \%$ or more, rapidly induced complete germination of both heat-activated and unheated spores of the Ames strain. There were no detectable differences in the rates of germination in this medium between spores preincubated with or without immune serum (data not shown). Heat-activated or unheated Ames spores pre-treated with water or preimmune sera and then incubated in $1 \%$ 

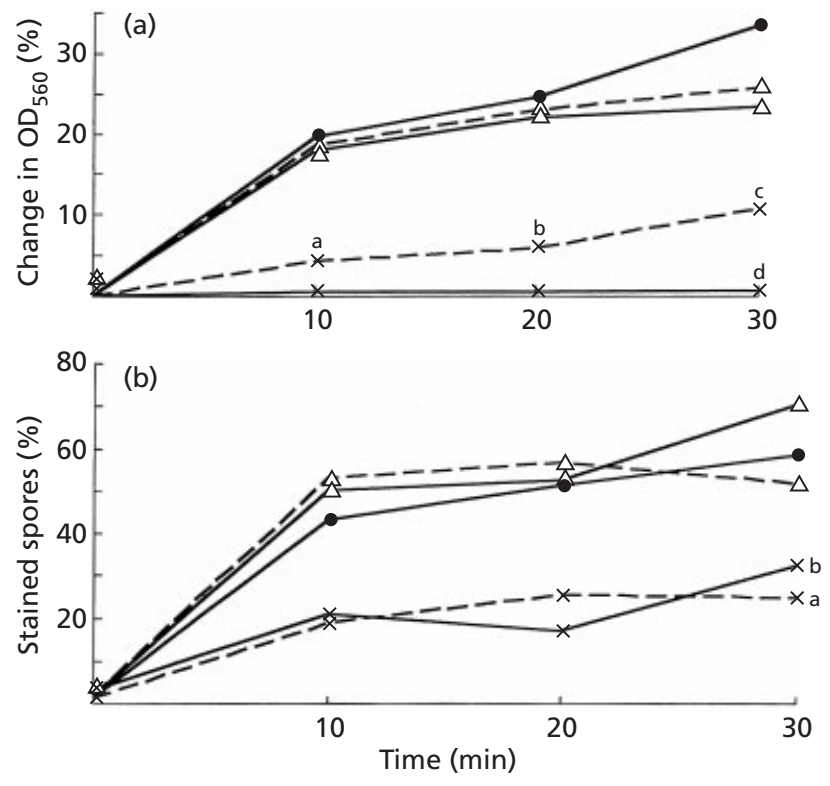

Fig. 2. In vitro germination of antibody-treated spores in $1 \%$ (v/v) BHI: (a) Percentage change in $\mathrm{OD}_{560}$ of Ames-H spores treated with dilutions of the rabbit anti-rPA antiserum $(X)$, preimmune serum $(\triangle)$, or water alone $(O)$ and incubated at $30{ }^{\circ} \mathrm{C}$ in $1 \% \mathrm{BHI}$. Samples were removed at intervals to assess the extent of germination by the decline (percentage change) in the $\mathrm{OD}_{560}$. The mean values of data obtained with sera diluted $1 / 100$ (dashed lines, four experiments) or 1/400 (solid lines, two experiments) are shown. ${ }^{a} P=0.03$ vs the preimmune serum $(1 / 100) .{ }^{b} P=0.036$ vs the preimmune serum $(1 / 100)$. 'Not significantly less than the corresponding value for the preimmune serum. ${ }^{\mathrm{d}}$ The mean values shown for the samples diluted 1/400 were not evaluated statistically. (b) Percentage stained spores of Ames-H. Spores were pre-treated with the dilutions of the rabbit anti-rPA antiserum, preimmune serum, or water alone and incubated as described in (a), except germination was assayed by determining the percentage of spores stained with crystal violet or methylene blue. Symbols are as described in (a). The mean values of data from four experiments are shown. ${ }^{a} P=0.016(10 \mathrm{~min}), 0.026(20 \mathrm{~min})$ and $0.004(30 \mathrm{~min})$ vs the corresponding values for the preimmune serum (1/100). The anti-PA (1/100) values for all three times were also less $(P<0.05)$ than the corresponding values for the samples incubated in $1 \% \mathrm{BHI}$ alone ('water'). ${ }^{\mathrm{b}} P=0.035$ (10 $\mathrm{min}), 0.018(20 \mathrm{~min})$ and $0.004(30 \mathrm{~min})$ vs the corresponding values for the preimmune serum (1/400). The anti-PA $(1 / 400)$ values for all three times were also less $(P<0.05)$ than the corresponding values for the samples incubated in $1 \% \mathrm{BHI}$ alone.

BHI exhibited up to $80 \%$ germination after $30 \mathrm{~min}$ at $30{ }^{\circ} \mathrm{C}$. Most of the spores that germinated did so within $10 \mathrm{~min}$. In contrast, pretreatment of spores with rabbit anti-rPA serum, purified rabbit anti-PA IgG and monkey anti-AVA sera was associated with the inhibition of spore germination (Fig. 2 and data not shown). Anti-PAor anti-AVA-treated spores demonstrated significantly smaller changes (percentage decrease) in $\mathrm{OD}_{560}$ (Fig. 2a) and fewer stained spores than did the samples pretreated with preimmune serum or water alone (Fig. 2b).

We investigated the possibility that Abs to the LF component of anthrax lethal toxin had a synergistic effect with anti-PA in inhibiting germination. In contrast to a previous report (Stepanov et al., 1996), neither whole antisera to recombinant LF nor the purified antiLF IgG had significant inhibitory activity alone and neither augmented the inhibition of germination observed with anti-PA Abs alone.

\section{Effect of anti-PA antiserum on loss of heat resistance during spore germination}

When germination was assayed by the decrease in $\mathrm{OD}_{560}$ and increase in staining, the effect of pretreating spores with sera as measured by one assay was mirrored by a comparable change in the other assay. Different results were obtained when germination was measured by the loss of heat resistance. As expected, a higher percentage of spores treated with preimmune serum $(>67 \%)$ than of anti-PA-treated spores $(<30 \%)$ became stainable within 5 min of incubation in $1 \%$ BHI. However, no significant differences in resistance to killing of spores by heat were conferred by pretreatment with anti-rPA compared to preimmune serum (data not shown). Regardless of pretreatment, the loss of heat-resistance began within seconds, i.e. the viability after heating of samples collected immediately after transfer to BHI decreased by 1.5 - to almost 10 -fold compared to paired unheated samples. The basis of the apparent inability of anti-PA Abs to inhibit germination as assayed by changes in heat resistance is not known. Perhaps, as described for Bacillus megaterium (Levinson \& Hyatt, 1966), Ames strain spores lose heat resistance first upon exposure to germinant, before the germination-inhibitory activity of anti-PA can have an effect.

\section{Immunoelectron microscopy}

The phagocytosis-enhancing and germination inhibition effects of sera containing anti-PA Abs suggested that PA or an antigenically similar protein might be present on the surface of the ungerminated spores. To detect antiPA reactivity, activated spores of Ames strain were incubated with rabbit anti-rPA $\mathrm{Ab}$ or preimmune serum and PA was detected with goat anti-rabbit IgG conjugated to gold particles. As shown in Fig. 3, spores preincubated with the anti-PA Ab (Fig. 3a) bound significantly more gold particles than those treated with the preimmune serum (Fig. 3b). There were no significant differences in the extent of binding of the Abs by germinated and ungerminated spores, as determined by counting the gold particles on the spore surfaces (data not shown).

\section{Spore extraction}

To begin characterizing the spore-associated PA activity, we extracted proteins from heat-activated spores. Several procedures described previously for other bacilli and clostridia were used in efforts to extract proteins from intact spores of $B$. anthracis. Extraction of the Ames strain with reagents containing guanidine or urea yielded a small to moderate amount of protein, $<20 \mu \mathrm{g} \mathrm{ml}^{-1}$ and $<1 \mathrm{mg} \mathrm{ml}^{-1}$, respectively (Ezzell \& Abshire, 1988; Gombas \& Labbe, 1981). When spores 

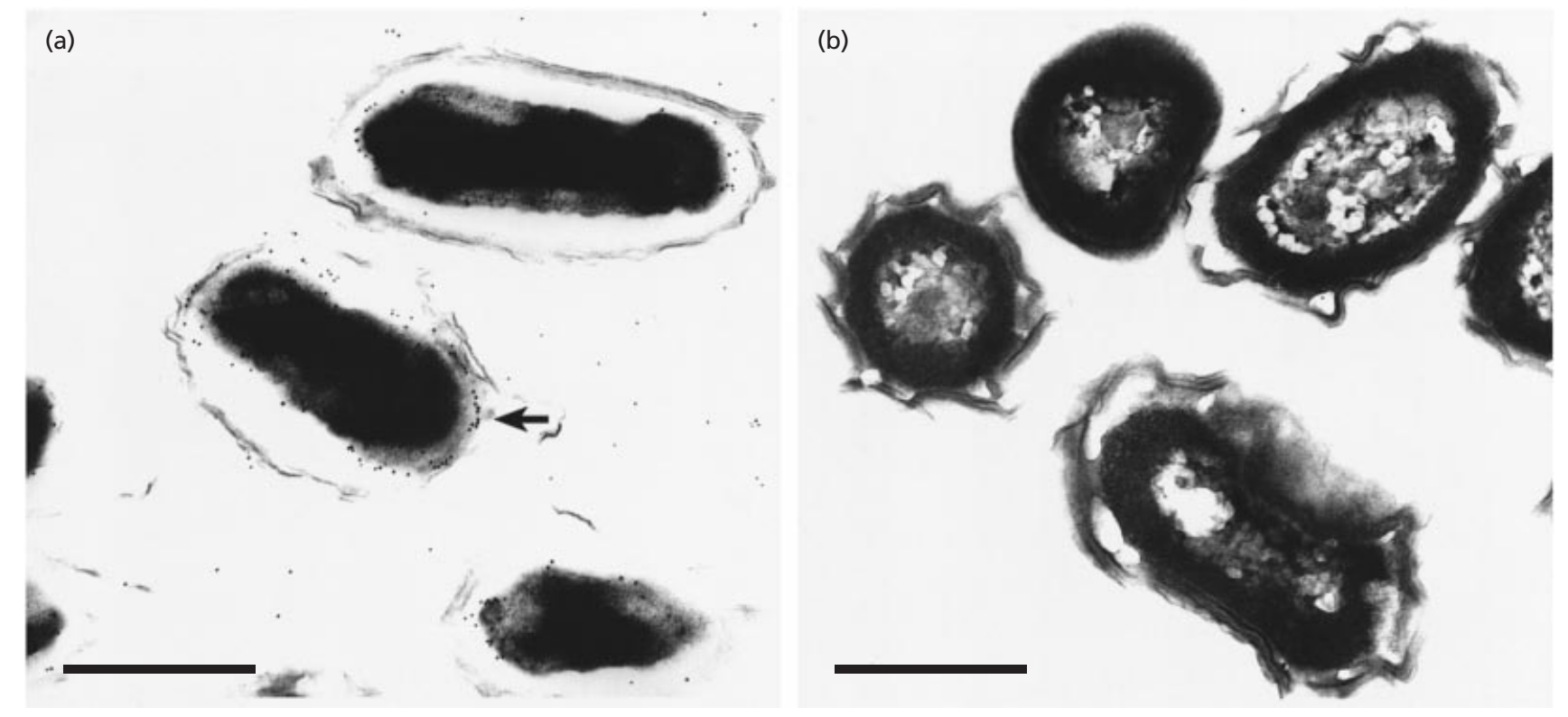

Fig. 3. Detection of PA associated with spores by immunogold labelling and electron microscopy. Ames-H spores were incubated with a $1 / 500$ dilution of rabbit anti-rPA antiserum (a) or preimmmune serum (b) and PA was detected with goat anti-rabbit IgG conjugated to $10 \mathrm{~nm}$ gold particles. Arrow shows the frequently observed peripheral location of the gold in Ames-H spores. Bars, $500 \mathrm{~nm}$.

were incubated with an alkaline solution consisting of $0 \cdot 1 \mathrm{M} \mathrm{DTT}, 0 \cdot 5 \%(\mathrm{w} / \mathrm{v})$ SDS and $0 \cdot 1 \mathrm{M} \mathrm{NaCl}, \mathrm{pH} 10 \cdot 0$ (DTT/SDS/ $\mathrm{NaCl})$, maximal protein yields of a few $\mathrm{mg} \mathrm{ml}^{-1}$ of extracted protein were obtained. This reagent was described previously for the extraction of spore coats of B. megaterium (Vary, 1973), and is similar to that described previously for extraction of spore coats of Bacillus cereus (Aronson \& Fitz-James, 1976; Beaman et al., 1971; DesRosier \& Lara, 1984).

\section{Gel electrophoresis and immunoblot analysis}

Proteins were extracted from intact spores with the DTT/SDS/ $\mathrm{NaCl}$ extraction reagent and analysed by SDS-PAGE and immunoblotting. We observed a large number of proteins on silver-stained SDS-PAGE gels (data not shown). However, only extracts of the PAproducing strains yielded a band on the immunoblot recognized by the affinity-purified rabbit anti-rPA IgG and equal in size to the $83 \mathrm{kDa}$ PA standard. The yield of the $83 \mathrm{kDa}$ anti-PA-reactive material from the spore surface appeared to vary with the stage of germination of the spores at the time of extraction and with the strain. Extracts from germinated spores of Sterne, Ames, ANR-1 (the toxigenic unencapsulated derivative of Ames) and the $\mathrm{PA}^{+} \mathrm{LF}^{-}$Sterne mutant RP42 reacted with anti-PA IgG on the blots (Fig. 4a, lanes 3, 5, 7 and 8 , respectively). Extracts prepared from ungerminated spores from the same batches did not (data not shown). The extracts from the germinated spores of the $\mathrm{PA}^{+}$and $\mathrm{PA}^{-}$strains also frequently yielded bands recognized by the rabbit anti-rPA IgG that did not co-migrate with $83 \mathrm{kDa}$ PA (Fig. 4a, lanes 3-9). Some may represent proteolytic fragments of $\mathrm{PA}$ (in $\mathrm{PA}^{+}$strains); others may be the same as the entities recognized by anti-PA $\mathrm{Ab}$ in the extract from $\Delta$ Ames (Fig. $4 a$, lane 6), a $\mathrm{PA}^{-}$pX01 derivative of Ames strain (Fig. 4a, lane 6), and might represent cross-reacting proteins.

Extracts of $\Delta$ Ames and RP4 $\left(\mathrm{LF}^{+}, \mathrm{PA}^{-}\right)$strains had no detectable band with the same mobility as $83 \mathrm{kDa} P A$ (Fig. 4a, lanes 6 and 9, respectively). Vollum1B (Fig. 4a, lane 4) was the only $\mathrm{PA}^{+}$strain that failed to produce a detectable anti-PA-reactive $83 \mathrm{kDa}$ band. Although the reason for the absence of PA from the Vollum 1B extract is unknown, this strain appeared to germinate more slowly and could thus differ in its kinetics of germination and synthesis of the $83 \mathrm{kDa}$ antigen compared to Ames or Sterne strains. Alternately, its PA-reactive material may have been localized at a site less accessible by our extraction procedure.

When the immunoblot of extracts was developed using a mixture of PA mAbs, the results verified the presence of $83 \mathrm{kDa}$ PA (Fig. 4b, lanes 3, 5, 7 and 8). It was present in low concentration in RP42 and appears as a light, $83 \mathrm{kDa}$ band (Fig. 4a, b, lane 8).

\section{DISCUSSION}

Upon inhalation of spores of $B$. anthracis by a susceptible host, the spores appear to be taken up locally by phagocytic cells. They are cleared or are carried to the regional lymph nodes where the surviving spores germinate and outgrow (Ross, 1957; Metchnikoff, 1905; Barnes, 1947; Lincoln et al., 1961; Guidi-Rontani et al., 1999; Hanna \& Ireland, 1999). The vegetative bacilli proliferate, spread systemically and secrete the anthrax toxins. An anti-spore immune response that interferes 

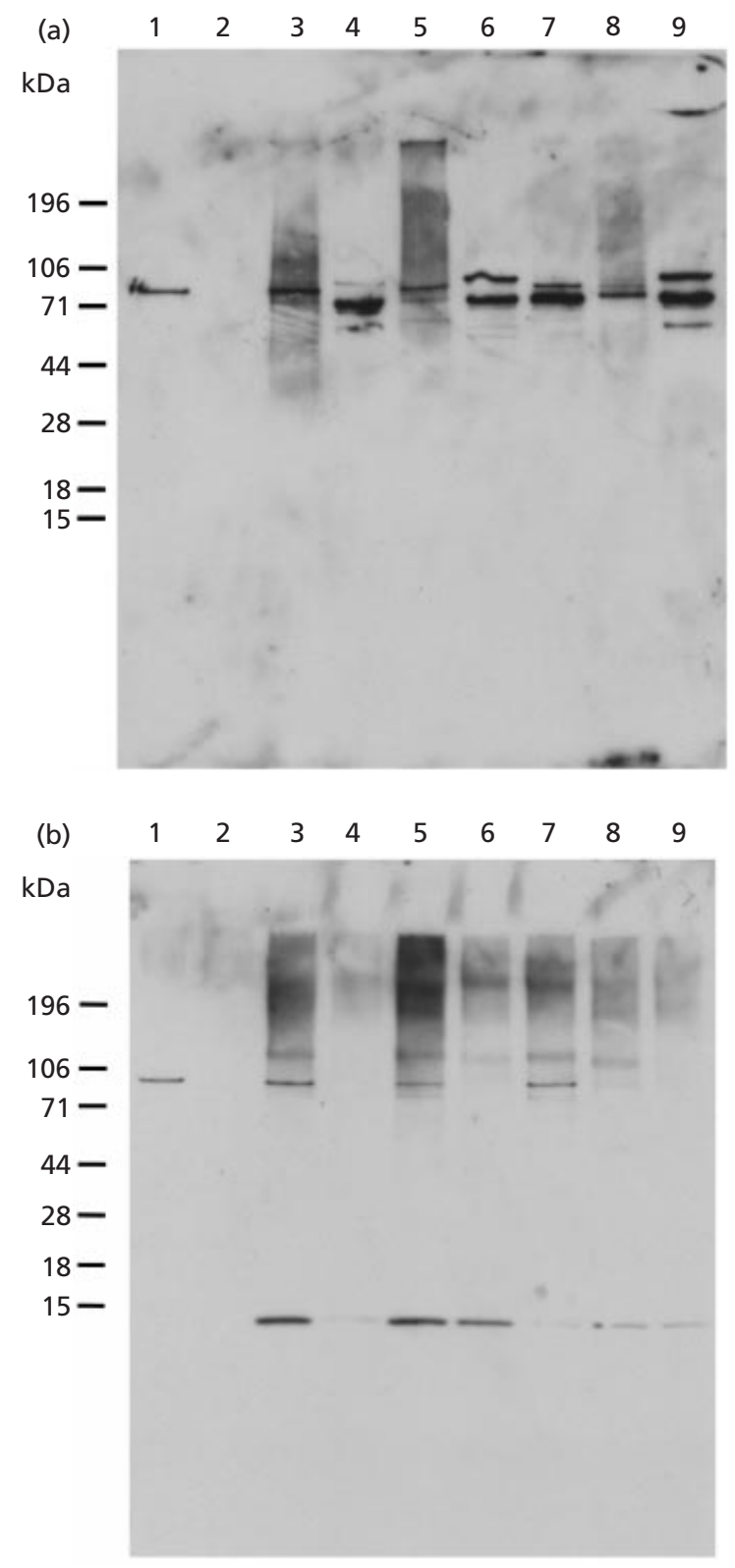

Fig. 4. Detection of proteins recognized by rabbit anti-rPA IgG and mouse anti-PA mAbs in spore surface extracts. Proteins were extracted from spores with the DTT/SDS/ $\mathrm{NaCl}$ extraction reagent and analysed by SDS-PAGE and immunoblotting. Proteins recognized by the Abs were detected by either HRPconjugated goat anti-rabbit IgG serum or HRP-conjugated goat anti-mouse IgG serum and enhanced chemiluminescence detection (Pierce). The numbers on the left side of the panels identify the positions of molecular mass standards. For each strain analysed, a spore preparation was made, heat-activated and divided into two samples. One was germinated in $\mathrm{BHI}$ and the other aliquot was left ungerminated. Protein extracts were prepared of both the germinated and ungerminated spores (data not shown; see Results). (a) Extracts from germinated spores were analysed using a PA affinity column-purified IgG prepared from the rabbit anti-rPA serum and the results are shown as follows: lane 1, purified PA; lane 2, blank; lane 3, Sterne; lane 4, Vollum1B; lane 5 , Ames; lane $6, \Delta$ Ames-1; lane 7, ANR-1; lane 8, RP42; and lane 9, RP4. (b) Extracts from germinated spores were also analysed by using a mixture of two mAbs to PA, made as described in the text. Lanes: same as in (a). early in this sequence of events, before the onset of bacteraemia and toxaemia, could conceivably offer effective protection against lethal infection.

Protection can be induced by vaccination with live vaccine strains or component vaccines containing PA such as AVA (Ivins et al., 1996; Pitt et al., 1996, 1999). Recent studies suggest that the titre of anti-PA $\mathrm{Ab}$ in immune animals correlates with protection (Pitt et al., 1999, unpublished data). Rabbits and monkeys vaccinated with AVA and challenged with virulent strains of $B$. anthracis only occasionally become detectably bacteraemic (Pitt et al., 1996; B. Ivins \& P. Fellows, unpublished data). The mechanism of protection against the early stages of infection afforded by AVA and other PA-based vaccines and the role of the antitoxin immune response are not fully elucidated.

Stepanov et al. (1996) reported that sera from animals vaccinated with an unencapsulated, toxin-producing, live vaccine strain had activities directed against the spores which may be important during the early stages of infection. Specifically, (1) germination was reported to be inhibited when the spores were incubated with antitoxin Abs (IgG fraction); and (2) the phagocytosis of spores by macrophages was stimulated by immune sera (IgM fraction) from animals vaccinated with the live spore vaccine. Our objectives were to develop in vitro systems for assaying the anti-spore activities of immune sera and to characterize these activities further using various antibody preparations. In agreement with the observations of Stepanov et al. (1996), made by using rabbit peritoneal macrophages and spores of $B$. anthra$c i s$, we found that sera from immune animals enhanced phagocytosis by murine peritoneal macrophages. The uptake of spores of both the fully virulent Ames strain and the $\mathrm{pX} 02^{-}$Sterne vaccine strain was enhanced by preincubating spores with antisera to purified PA (Fig. 1). Stepanov et al. (1996) indicated that rabbit antianthrax sera promoted spore phagocytosis and we observed comparable activity in the antisera from this species (Fig. 1a, c) and from other species, such as monkeys vaccinated with AVA (Fig. 1b). Although phagocytosis was attributed primarily to IgM Abs by Stepanov et al. (1996), we showed that anti-PA IgG Abs had significant activity.

The stage of germination when $B$. anthracis initiates synthesis of the toxin components, and when the spores become amenable to anti-PA stimulated phagocytosis, is not known. The spores do not germinate in the culture medium alone (DMEM) used in the macrophage phagocytosis experiments. However, the addition of animal sera from some species supports germination; e.g. the addition of fetal bovine serum, but not of horse or mouse serum, to DMEM stimulates germination of Ames spores (S. Welkos, unpublished data). Regardless of this, we observed that spores that are ungerminated (refractile) were phagocytosed at least as well as those that had completely germinated prior to phagocytosis (data not shown). Also, we demonstrated by immunoelectron microscopy the presence of an anti-PA reactive entity on the surface of ungerminated spores. We are 
currently pursuing studies to determine the kinetics of expression of the PA-reactive substrate during the process of germination and outgrowth.

Phagocytosis of the infecting spore appears to be a major step in the pathogenesis of inhalational anthrax, as reviewed recently (Dixon et al., 1999; Hanna \& Ireland, 1999). Ross (1957) showed that inhaled spores were phagocytosed by alveolar cells and were either cleared locally or were transported within the macrophages to the regional lymphatics where they germinated and outgrew. Guidi-Rontani et al. (1999) showed more recently that alveolar macrophages from infected mice can similarly phagocytose spores of B. anthracis and that the spores germinate within the phagocyte. Germination of the spores upon phagocytosis in vitro by peritoneal macrophages or macrophage-like tissue culture cells has also been demonstrated (Dixon et al., 1999; Hanna \& Ireland, 1999; S. Welkos and others, unpublished data). The subsequent fate of the germinated spores is not well defined. Scenarios depicting both the outgrowth and release of the organisms (Hanna \& Ireland, 1999; Dixon et al., 2000; J. Ireland, unpublished data) and the loss of viability of the phagocytosed spores (Guidi-Rontani et al., 1999; Welkos et al., 1989; S. Welkos and others, unpublished data) have been reported.

An antitoxin-mediated enhancement of phagocytosis could potentially contribute to the protection from infection induced by AVA vaccination. In recent studies on the effect of anti-PA Abs on the uptake and fate of phagocytosed spores, we observed that treating spores prior to phagocytosis was associated with an increased extent of intracellular germination and subsequent enhanced rate of killing (S. Welkos and others, unpublished data). The induction of such anti-spore activities in the vaccinated host might contribute to a protective immune response.

As reported by Stepanov et al. (1996), another anti-spore activity associated with antitoxin antisera was the inhibitory effect on spore germination. Ames strain spores pre-treated with rabbit anti-rPA antiserum exhibited a significantly smaller decrease in $\mathrm{OD}_{560}$ and fewer stained spores after incubation than did the control samples (Fig. 2a, b and data not shown); similar differences were observed when germination was assessed by the loss of refractivity, but not by the loss of heat resistance (data not shown). The inhibition of germination could potentially be another Ab-mediated protective mechanism. If extracellular germinants are present at the inoculation site, the spores might be able to germinate and the bacilli multiply outside the macrophage. Abs directed to the spore might retard this process until activated macrophages arrive and phagocytose the spores. Further in vivo studies to define the sites (i.e. inside or outside of phagocytes) and stimulants of spore germination in the host are needed.

We are characterizing the entity on the surface of spores that is the target of the anti-spore immunity. It appears to be homologous antigenically to PA, but could be a variant of the toxin protein or a different cross-reactive surface protein. There also may be more than one target. We identified potential candidates by gel electrophoresis and immunoblotting of spore extracts. Extracts of germinated spores from all PA-producing strains except Vollum1B reacted with anti-PA Abs on immunoblots (Fig. 4); and they each produced an $83 \mathrm{kDa}$ protein recognized by mAbs to PA (Fig. 4b). In contrast to results using germinated spores, extracts from ungerminated spores from the same batches did not contain a detectable anti-PA reactive protein (data not shown). However, when binding of anti-PA Abs by spores was studied by electron microscopy (Fig. 3), there were no detectable differences in the extent of binding of the Abs by germinated and ungerminated spores. Thus, although anti-PA-reactive material appears to be present in both germinated and ungerminated spores, it appears to be easier to extract or present in higher concentration in the former compared to the latter spore stage. The procedure used for spore extraction is optimal for extracting the spore coat proteins from several bacilli (Vary, 1973; Aronson \& Fitz-James, 1976) and also solubilizes exosporium preparations (Beaman et al., 1971; DesRosier \& Lara, 1984). In addition to the $83 \mathrm{kDa}$ PA, other entities appeared to be recognized by the anti-rPA antiserum in immunoblots. Thus, further studies are being performed to elucidate several parameters, including: (1) the kinetics of PA expression in anthrax spores; (2) the localization of PA in the outer spore surfaces (i.e. the spore coats and the exosporium); and (3) the identities of all anti-PA-reactive antigens.

In summary, antisera to PA had an anti-spore effect(s) that was detectable by at least two activities: (1) the stimulation of phagocytosis by macrophages of spores of the Ames and Sterne strains; and (2) the inhibition of spore germination in $1 \%$ BHI. Activity was shown with rabbit antisera to recombinant and non-recombinant $\mathrm{PA}, \mathrm{IgG}$ purified from rabbit antisera to PA, and monkey antisera to the human AVA vaccine. PA or PA-like antigen was detected by electron microscopy on immunogold-labelled spores, and proteins extracted from spores reacted with anti-PA IgG and mAbs on Western blots. It is conceivable that the anti-spore effect(s) of antitoxin antibodies in individuals vaccinated against anthrax might be protective early in infection before outgrowth and toxin secretion by bacilli. Our goals are to identify the genes encoding the spore proteins reacting with anti-PA Abs and to determine the role of the antitoxin-mediated anti-spore activity in early protection against infection.

\section{ACKNOWLEDGEMENTS}

We thank M. Mock and co-workers for kindly providing strains RP4 and RP42; J. Weeks, P. Merrill and K. Kuhn for excellent technical assistance; and S. Tobery for preparation of peritoneal macrophages.

\section{REFERENCES}

Aronson, A. I. \& Fitz-James, P. (1976). Structure and morphogenesis of the bacterial spore coat. Bacteriol Rev 40, 360-402. 
Barnes, J. M. (1947). The development of anthrax following the administration of spores by inhalation. $\mathrm{Br} J$ Exp Pathol 28, 385.

Beaman, T. C., Pankratz, H. S. \& Gerhardt, P. (1971). Paracrystalline sheets reaggregated from solubilised exosporium of Bacillus cereus. J Bacteriol 107, 320-324.

DesRosier, J. R. \& Lara, J. C. (1984). Synthesis of the exosporium during sporulation of Bacillus cereus. J Gen Microbiol 103, 935-940.

Dixon, T. C., Meselson, M., Guillemin, J. \& Hanna, P. C. (1999). Anthrax. N Engl J Med 341, 815-826.

Dixon, T. C., Fadl, A. A., Koehler, T. M., Swanson, J. A. \& Hanna, P. C. (2000). Early Bacillus anthracis-macrophage interactions: intracellular survival and escape. Cell Microbiol 2, 453-463.

Ezzell, J. W., Jr \& Abshire, T. G. (1988). Immunological analysis of cell-associated antigens of Bacillus anthracis. Infect Immun 56, 349-356.

Ezzell, J. S., Abshire, T. G. \& Little, S. F. (1990). Identification of Bacillus anthracis by using monoclonal antibody to cell wall galactose- $\mathrm{N}$-acetylglucosamine polysaccharide. J Clin Microbiol 28, 223-231.

Fritz, D. L., Jaax, N. K., Lawrence, W. B., Davis, K. J., Pitt, M. L. M., Ezzell, J. W. \& Friedlander, A. M. (1995). Pathology of experimental inhalation anthrax in the rhesus monkey. Lab Invest 73, 691-702.

Gombas, D. \& Labbe, R. (1981). Extraction of spore-lytic enzyme from Clostridium perfringens spores. J Gen Microbiol 126, 37-44.

Guidi-Rontani, C., Weber-Levy, M., Labruyere, E. \& Mock, M. (1999). Germination of Bacillus anthracis spores within alveolar macrophages. Mol Microbiol 31, 9-17.

Hanna, P. C. \& Ireland, J. A. W. (1999). Understanding Bacillus anthracis pathogenesis. Trends Microbiol 7, 180-182.

Hansen, M. B., Nielsen, S. \& Berg, K. (1989). Re-examination and further development of a precise and rapid dye method for measuring cell growth/cell kill. J Immunol Methods 119, 203-210.

Ivins, B. E. \& Welkos, S. L. (1986). Cloning and expression of the Bacillus anthracis protective antigen gene in Bacillus subtilis. Infect Immun 54, 537-542.

Ivins, B. E., Fellows, P. F., Pitt, M. L. M., Estep, J. E., Welkos, S. L., Worsham, P. A. \& Friedlander, A. M. (1996). Efficacy of a standard human anthrax vaccine against Bacillus anthracis aerosol spore challenge in rhesus monkeys. Salisbury Med Bull Suppl 87, $125-126$.

Leighton, T. J. \& Doi, R. H. (1971). The stability of messenger ribonucleic acid during sporulation in Bacillus subtilis. J Biol Chem 246, 3189-3195.

Levinson, H. S. \& Hyatt, M. T. (1966). Sequence of events during Bacillus megaterium spore germination. J Bacteriol 91, 18111818.

Lincoln, R. E., Rhian, M. A., Klein, F. \& Fernelius, A. (1961). Pathogenesis as related to the physiological state of anthrax spores and cell. In Spores II, pp. 255-272. Edited by H. O. Halvorson. Minneapolis, MN: Burgess Publishing Co.

Little, S. F. \& Knudson, G. B. (1986). Comparative efficacy of Bacillus anthracis live spore vaccine and protective antigen vaccine against anthrax in the guinea pig. Infect Immun 52, $509-512$
Little, S. F. \& Lowe, J. R. (1991). Location of receptor-binding region of protective antigen from Bacillus anthracis. Biochem Biophys Res Commun 180, 531-537.

Little, S. F., Leppla, S. H. \& Cora, E. (1988). Production and characterization of monoclonal antibodies to the protective antigen component of Bacillus anthracis toxin. Infect Immun 56, 1807-1813.

Little, S. F., Novak, J. M., Lowe, J. R., Leppla, S. H., Singh, Y., Klimpel, K. R., Lidgerding, B. C. \& Friedlander, A. M. (1996). Characterization of lethal factor binding and cell receptor binding domains of protective antigen of Bacillus anthracis using monoclonal antibodies. Infect Immun 56, 1807-1813.

Little, S. F., Ivins, B. E., Fellows, P. F. \& Friedlander, A. M. (1997). Passive protection by polyclonal antibodies against Bacillus anthracis infection in guinea pigs. Infect Immun 65, 5171-5175.

Metchnikoff, E. (1905). Immunity in Infective Diseases. Cambridge: Cambridge University Press.

Pepper, D. S. (1990). A user's guide to protein A. In Laboratory Methods in Immunology, vol. II, pp. 169-180. Edited by H. Zola. Boca Raton, FL: CRC Press.

Pezard, C., Berche, P. \& Mock, M. (1991). Contribution of individual toxin components to virulence of Bacillus anthracis. Infect Immun 59, 3472-3477.

Pezard, C., Duflot, E. \& Mock, M. (1993). Construction of Bacillus anthracis mutant strains producing a single toxin component. $J$ Gen Microbiol 139, 2459-2463.

Pitt, M. L. M., Ivins, B. E., Estep, J. E., Farchaus, J. \& Friedlander, A. M. (1996). Comparison of the efficacy of purified protective antigen and MDPH to protect non-human primates from inhalation anthrax. Salisbury Med Bull Suppl 87, 130.

Pitt, M. L. M., Little, S., Ivins, B. E., Fellows, P. F., Boles, J., Barth, J., Hewetson, J. H. \& Friedlander, A. M. (1999). In vitro correlate of immunity in an animal model of inhalational anthrax. $J A p p l$ Microbiol 87, 304

Ross, J. M. (1957). The pathogenesis of anthrax following the administration of spores by the respiratory route. J Pathol Bacteriol 73, 485-494.

Stepanov, A. V., Marinin, L. I., Pomerantsev, A. P. \& Staritsin, N. A. (1996). Development of novel vaccines against anthrax in man. J Biotechnol 44, 155-160.

Towbin, H., Staehelin, T. \& Gordon, J. (1979). Electrophoretic transfer of proteins from polyacrylamide gels to nitrocellulose sheets: procedure and some applications. Proc Natl Acad Sci US A 76, 4350-4354.

Vary, J. (1973). Germination of Bacillus megaterium spores after various extraction procedures. J Bacteriol 116, 797-802.

Welkos, S. L., Trotter, R. W., Becker, D. M. \& Nelson, G. O. (1989). Resistance to the Sterne strain of B. anthracis: phagocytic cell responses of resistant and susceptible mice. Microb Pathog 7, $15-36$.

Worsham, P. L. \& Sowers, M. R. (1999). Isolation of an asporogenic (spoOA) protective antigen-producing strain of Bacillus anthracis. Can J Microbiol 45, 1-8.

Received 24 August 2000; revised 3 January 2001; accepted 29 January 2001. 